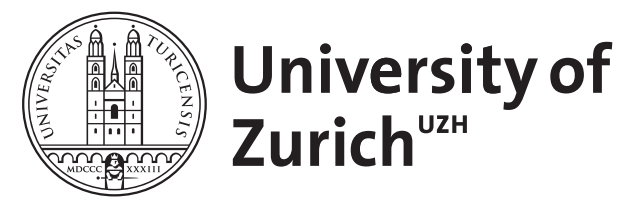

\title{
A cost of cryptic female choice in the yellow dung fly
}

\author{
Ward, Paul I ; Wilson, Alastair J ; Reim, Constanze
}

\begin{abstract}
Female dung flies Scathophaga stercoraria (L.) store sperm from several males in three or four spermathecae. Selection on the number of spermathecae was successful and the morphological intermediate stages in the evolution from three to four spermathecae are illustrated. The genetic quality of a male from a female's perspective depends on an interaction between their genotypes and the microhabitat in which the offspring will grow. Females influence the paternity pattern of their offspring, and do this differently in different microhabitats. Females with four spermathecae are better able to influence paternity than are those with three spermathecae. However, there must be a cost to building and maintaining an extra spermatheca. We estimate, using the animal model on pedigree data, that this cost is approximately five eggs per clutch, i.e. around $8 \%$ of the mean clutch size. This is a substantial cost and such costs should not be ignored in discussions of the benefits to females of assessing the genetic qualities of their mating partners. We suggest that the number of spermathecae in the study population is stable because the relative benefits in quality of offspring through cryptic female choice is balanced by the costs in total numbers of offspring.
\end{abstract}

DOI: https://doi.org/10.1007/s10709-007-9205-y

Posted at the Zurich Open Repository and Archive, University of Zurich

ZORA URL: https://doi.org/10.5167/uzh-4921

Journal Article

Published Version

Originally published at:

Ward, Paul I; Wilson, Alastair J; Reim, Constanze (2008). A cost of cryptic female choice in the yellow dung fly. Genetica, 134(1):63-67.

DOI: https://doi.org/10.1007/s10709-007-9205-y 


\title{
A cost of cryptic female choice in the yellow dung fly
}

\author{
Paul I. Ward · Alastair J. Wilson · Constanze Reim
}

Received: 26 February 2007/ Accepted: 1 August 2007/Published online: 30 September 2007

(C) Springer Science+Business Media B.V. 2007

\begin{abstract}
Female dung flies Scathophaga stercoraria (L.) store sperm from several males in three or four spermathecae. Selection on the number of spermathecae was successful and the morphological intermediate stages in the evolution from three to four spermathecae are illustrated. The genetic quality of a male from a female's perspective depends on an interaction between their genotypes and the microhabitat in which the offspring will grow. Females influence the paternity pattern of their offspring, and do this differently in different microhabitats. Females with four spermathecae are better able to influence paternity than are those with three spermathecae. However, there must be a cost to building and maintaining an extra spermatheca. We estimate, using the animal model on pedigree data, that this cost is approximately five eggs per clutch, i.e. around $8 \%$ of the mean clutch size. This is a substantial cost and such costs should not be ignored in discussions of the benefits to females of assessing the genetic qualities of their mating partners. We suggest that the number of spermathecae in the study population is stable because the relative benefits in quality of offspring through cryptic female choice is balanced by the costs in total numbers of offspring.
\end{abstract}

Keywords Antagonistic pleiotropy - Heritability · Postcopulatory selection - Selection experiment . Scatophaga $\cdot$ Sperm competition

P. I. Ward $(\bowtie) \cdot$ C. Reim

Zoological Museum, University of Zurich, Winterthurerstr. 190,

Zurich 8057, Switzerland

e-mail: pward@zm.unizh.ch

A. J. Wilson

Institute of Evolutionary Biology, University of Edinburgh, Edinburgh EH9 3JT, UK

\section{Introduction}

The females of many animal species mate with more than one male in a single reproductive bout (Arnqvist and Rowe 2005; Arnqvist and Nilsson 2000), making the opportunity for sperm competition (Parker 1970) and cryptic female choice (Eberhard 1996) common. Most discussions seeking a general explanation of female multiple mating focus on the potential benefits to the females, e.g. obtaining good genes from the highest quality males for their offspring or ensuring genetic compatibility between themselves and the male which fathers the offspring (Zeh and Zeh 2001). However, there must also be costs to making a choice, since mating itself is often costly and must at least take some time. Such costs must influence the evolution of female choice and, if substantial, could even prevent its evolution.

Females of many animal species store sperm within their bodies in sometimes elaborate specialised organs (Eberhard 1996). It is becoming clear that the sperm from different males may be stored or treated, at least to some extent, differently within female storage systems (e.g. Otronen et al. 1997; Beese and Baur 2006; Curril and LaMunyon 2006). Such a separation could give females substantial control over paternity when it comes to using the sperm to fertilise eggs. This is because sperm are typically released from storage in a controlled manner and a female could influence paternity by controlling from which part of her system sperm are released to move, or be moved, to the site of fertilization. The female could therefore adopt a mate-now-choose-later strategy of mate choice (see Ward, in press). The construction, maintenance and use of an elaborate sperm storage system rather than a simple one could represent a substantial cost of choice for females. 
The yellow dung fly Scathophaga stercoraria (L.) has long been a study animal for the examination of sperm competition (Parker 1978) and, more recently, for cryptic female choice (Ward 2000, in press). A female has either three or four spermathecae, the sperm storage organs, and can store the sperm from different males to some extent separately (Otronen et al. 1997). Females in the laboratory exert cryptic female choice amongst males at the phosphoglucomutase (PGM) locus, choosing males of different PGM genotype under different environmental conditions (Ward 2000). Females with four spermathecae are better able to do this, at least when they are large, than are females with three spermathecae. In the field, eggs of different PGM genotype are laid in different parts of a dung pat, in a pattern consistent with the lab results (Ward et al. 2002). Since larvae of different PGM genotypes grow best in variable or constant temperature conditions (Ward 1998), the choice benefits the females as she can, at least to some extent, match the genotypes of her offspring to the environmental conditions under which they will grow. This means that a female's offspring will be larger than would be the case with a random association between offspring genotype and larval growth environment. Large body size is of selective advantage to both male and female adult flies (Jann et al. 2000).

Most female yellow dung flies from the field have three spermathecae, with around 10\% having four (Ward 2000). Lines of flies were selected to have either three or four spermathecae, showing that the variation is at least partially genetic. In this paper the pedigree data from the selection lines is used to estimate the heritability of the variation. In addition, the heritabilities of the correlated responses in the numbers of spermathecal ducts and the numbers of eggs in a full first clutch are estimated.

\section{Materials and methods}

Lines of flies were bred to have either three or four spermathecae. For details of the breeding methods, see Ward (2000). Briefly, flies from a population from Fehraltdorf, Switzerland were maintained in individual containers from adult eclosion to sexual maturity. They were fed on water, sugar and live Drosophila melanogaster. Matings (at random but with no sib matings) were conducted in small bottles containing a small portion of dung. The parentage of each fly was recorded each generation. The number of eggs oviposited by each female was subsequently counted. Egg volume was not measured but varies little in females' first clutches, as used here, and is not related to a female's body size (Jann and Ward 1999). It is therefore extremely unlikely that egg volume affected the results presented here. After oviposition, the eggs were transferred to larger containers to avoid larval competition for food affecting body size during development. Each egg therefore had an equal chance of developing into an adult. Also after oviposition, each female was dissected, after being killed under $\mathrm{CO}_{2}$ anaesthetic, and the number and morphology of her spermathecae and the number of spermathecal ducts counted. It was also noted whether a female had laid all her eggs or if some were left in the abdomen.

Most females from the field have three spermathecae and a normal spermatheca is approximately globular (Hosken et al. 1999; Fig. 1a). The most common variant of a single spermatheca is a heart-shaped one (Fig. 1b). These variants appear to be a developmental transition to a doublet spermatheca and were used in the selection lines for four spermathecae (see below). If four spermathecae were present at the beginning of the breeding program, there a

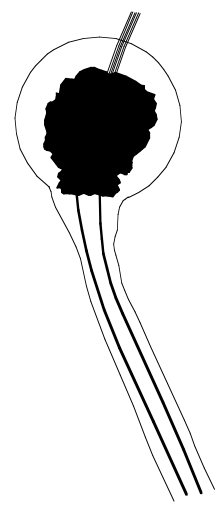

C

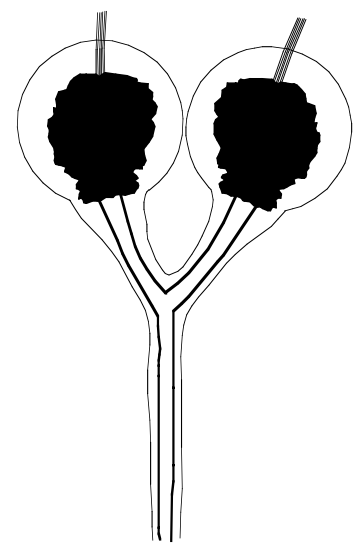

b

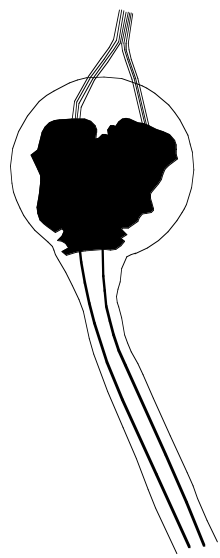

d

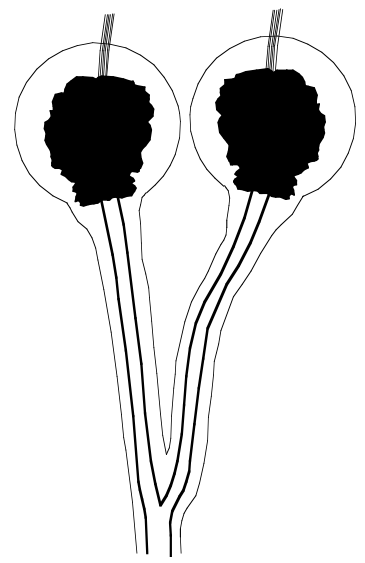

Fig. 1 The morphological stages in the development of two spermathecae from one. Each chitinized spermathecae (black) is surrounded by a largely glandular layer and has a muscle attached to a projection into the lumen of the spermatheca. This muscle probably aids filling and emptying of the spermatheca. The duct to the bursa copulatrix is surrounded by a layer of largely muscular tissue. This muscle is probably involved in the movement of sperm to the spermatheca 
were normally only three spermathecal ducts, with two spermathecae sharing a duct, a common pattern in other fly species. The point of the separation of the common duct from the bursa into a duct portion leading to each spermatheca also varied along its length (Fig. 1c, d).

The batches of eggs were raised to adulthood from the females with the appropriate morphology for their selection regime. The heart-shaped variants were treated as being 'four spermathecae' in those lines. The other batches of eggs were discarded before the adult flies emerged. No selection was directly exerted on the number of spermathecal ducts or on the numbers of eggs.

During the selection program one female was found with two spermathecae and one with five. However, neither of these females produced mature eggs.

Only females which had laid all their eggs were used in the estimations of the heritabilities below. This was done because in the field almost all females lay their full clutch. The laying of partial clutches is therefore almost certainly a lab artefact, probably of disturbance. It made no significant difference to any of the estimates.

After 15 generations the replicate lines produced only females with the appropriate number of spermathecae, at which point the program was stopped (Ward 2000). The lines within each selection regime were treated here as a single population. Only flies from the third generation onwards were used in the analyses, since this was the generation which would have been free of any maternal or environmental effects from the field. The realized heritabilities were estimated as twice the slopes of the cumulative responses to selection, since selection was only exerted on females (Roff et al. 1999). The heritabilities for the number of spermathecae, the number of spermathecal ducts and first clutch size were also estimated by restricted maximum likelihood using animal models implemented in the program ASReml. The animal model is a form of mixed effects model of additive genetic variance in the base (unselected) population, estimated from phenotypic data and the pedigree-derived matrix of relationships among individuals (for more details see Lynch and Walsh 1998; Kruuk 2004).

\section{Results}

As expected, there was a significant realized heritability for the number of spermathecae in the four-spermathecae selection regime (Table 1). The realized heritability for the number of spermathecae in the three spermathcae regime was not significantly different to zero. This is perhaps not surprising, as the field population already began with around $90 \%$ females with three spermathecae, meaning the change in mean number over the 15 generations was rather small. The total heritabilities for the numbers of spermathecae from the animal model followed the same pattern as the realized heritabilities, and were very similar, though this need not be the case (see Lynch and Walsh 1998).

There was also a significant correlated response in the four spermathecae regime for the number of spermathecal ducts. At generation 15 all the four spermathecae females also had four ducts to the bursa. Although it was not recorded in detail, the process by which the change from three to four ducts came about was that the junction point of the separation of the common duct to one portion going to each spermatheca was progressively towards the bursa at the generations went by (i.e. in the direction from Fig. 1c, d).

There was no significant change in the number of ducts, or significant heritability for the character, in the three spermathecae regime. This is again most likely because of the lack of variation in the founding population.

There was a significant correlated response in the numbers of eggs in a full first clutch in the three spermathecae regime. The numbers changed from approximately 54 per clutch to approximately 66 by the end of the program $(y=50.539+1.032 x( \pm 0.228, \beta=$ 0.179), $t=4.525, \quad P<0.001, \quad n=623$ (Fig. 2a). The numbers of eggs in the four spermathecae regime was remarkably constant at approximately 60 eggs per clutch $(y=58.249+0.183 x( \pm 0.217, \beta=0.0 .039), t=0.843$, $P=0.399, n=460$ (Fig. 2b). Correspondingly, the heritability of the numbers of eggs in this regime did not differ significantly from zero.
Table 1 The realized and total heritabilities of the numbers of spermathecae, spermathecal ducts and eggs

\begin{tabular}{lll}
\hline & & Realized heritability \\
\hline Number of spermathecae & 3 Spermathecae lines & $0.108 \pm 1.42, P=0.466$ \\
& 4 Spermathecae lines & $0.282 \pm 0.06, P=0.002$ \\
& & Total heritability (animal model) \\
Number of spermathecae & 3 Spermathecae lines & $0.127 \pm 0.07, P>0.05$ \\
Number of ducts & 4 Spermathecae lines & $0.271 \pm 0.08, P<0.001$ \\
& 3 Spermathecae lines & $0.060 \pm 0.05, P>0.05$ \\
Number of eggs & 4 Spermathecae lines & $0.170 \pm 0.07, P<0.02$ \\
& 3 Spermathecae lines & $0.145 \pm 0.07, P<0.05$ \\
& 4 Spermathecae lines & $0.060 \pm 0.09, P>0.05$ \\
\hline
\end{tabular}



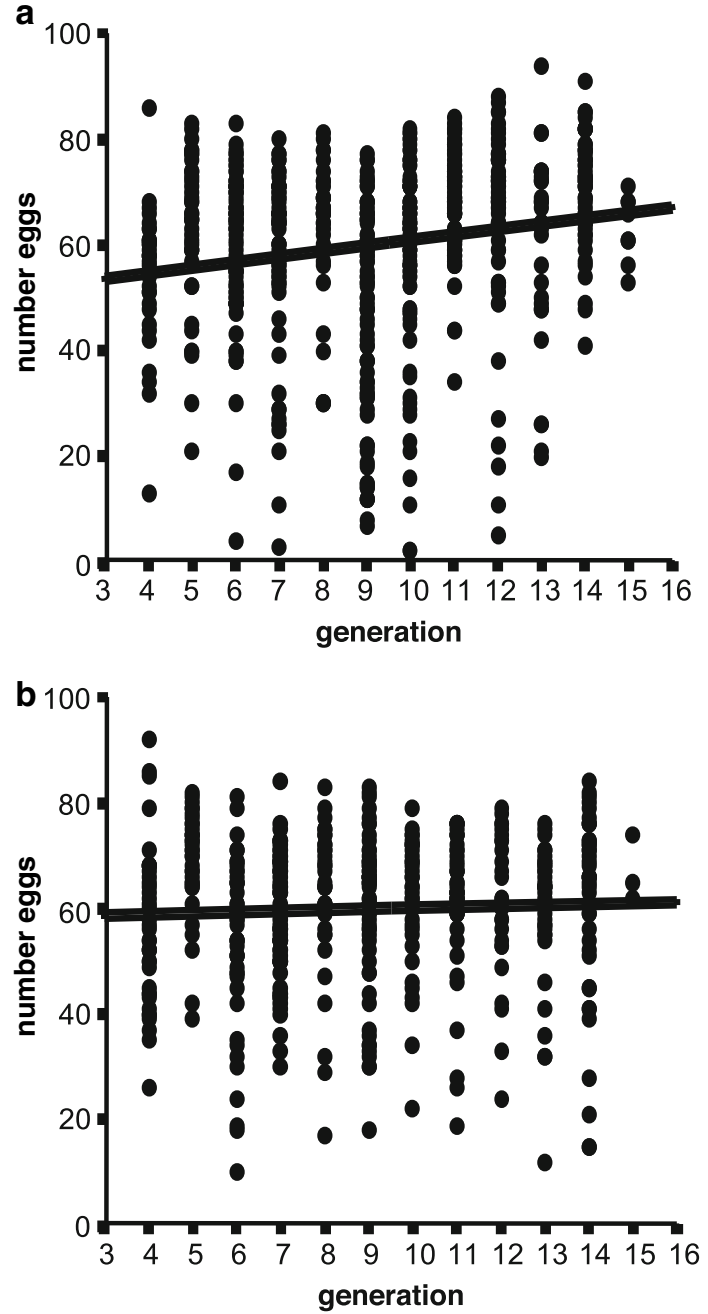

Fig. 2 The numbers of eggs laid by females in the two selection regimes. (a) The three spermathecae females and (b) the four spermathecae females. There was a significant increase in clutch sizes in the three spermathecae females over the experiment but no significant change in the clutch sizes of the four spermathecae ones

\section{Discussion}

There is clearly a cost in reproductive output for females which have four rather than three spermathecae. The clutch size of the four spermathecae females remained remarkably constant at approximately 60 eggs, while in the three spermathecae females there was an increase from approximately 54 to 66 eggs per clutch over the selection program. The smaller clutch size of the three spermathecae females at the beginning of the program could even be due to the alleles responsible for the development of the extra spermatheca having negative effects in normal females. These antagonistically pleiotropic alleles may in general also be recessive, as it took some generations to eliminate them from the three spermathecae lines. Their persistence in the population (see below) thus suggests they may be beneficial elsewhere in the female, or even male, life history (Rice et al. 2006). Alternatively, there may be linkage groups of genes for spermathecal morphology and clutch size with similar effects to the pleiotropies. Since the population sizes and generation numbers are not large, this possibility cannot be excluded.

The construction of an extra spermatheca, and its associated duct to the bursa, as well as other structures such as muscles and nerves, costs a normal female about $8 \%$ of her reproductive output per clutch, calculated as five eggs from 66 at generation 15 . Note however that as the clutch size could have increased even further in three spermathecae females had the experiment continued longer, this could be an underestimate of the cost. Nevertheless, it is a considerable cost, comparable to the approximately $13 \%$ reduction in clutch size from a female's first to her fourth clutch (Jann and Ward 1999). Such costs will clearly strongly influence the evolution of female choice mechanisms and should be considered in discussions of the phenomenon.

The genetic control of the development of the number of spermathecal ducts is considerably more complicated than would be suggested by a direct change from three to four spermathecae. The first stage of the observed process was that there was a single spermatheca, which appeared to have partially developed as two spermathecae but these had failed to separate during development, resulting in a heartshaped morphology. The next stage was that two spermathecae had very short duct portions leading to them from a junction in a single duct leaving the bursa. The point of the junction moved down the common duct in the course of selection, although no direct selection was applied to this character. It seems intuitively likely that a female with a separate duct to each spermatheca could better separate the sperm from different males than could a female with spermathecae on a common duct. This is because there does not appear to be any physical barrier, such as a valve, to prevent sperm actively moving in either direction when they reach the junction. Although the mass movement of sperm through the ducts is probably due to female muscular contractions (Hosken and Ward 2000), sperm are themselves certainly motile and could well be able to make the small adjustments necessary to move to either side of a branch. It should be noted that the large females which could better control paternity in the lab all had four spermathecae and four ducts (Ward 2000).

Females from the field site have had predominantly three spermathecae since samples have been taken in 1989 (Ward P. I., personal observation). Four spermathecae or the heartshaped singlet variant have been the only other morphologies repeatedly found. Although one female with two and one with five spermathecae were found during the selection program, neither produced mature eggs. This suggests that 
there is strong stabilizing selection for the number of spermathecae, with a lack of substantial genetic variation for fewer or for more. This leads us to speculate that there must be a balance between the higher mean quality of offspring produced by females with four spermathecae, because of the better fit between offspring genotype and the environmental conditions in which each egg is laid and/or in which the larva will have to grow, and the greater number of offspring produced by females with three spermathecae. The identification and quantification of such trade-offs in other species would certainly be of interest.

Acknowledgements Thanks to Wolf Blanckenhorn for his comments on this material, to Trevor Pitcher for organising the seminar and him and two anonymous referees for comments and to Yves Choffat for preparing the figures. AJW is funded by the Natural Environment Research Council.

\section{References}

Arnqvist G, Nilsson T (2000) The evolution of polyandry: multiple mating and female fitness in insects. Anim Behav 60:145-164

Arnqvist G, Rowe L (2005) Sexual conflict. Princeton University Press, Princeton

Beese K, Baur B (2006) Expandable spermatheca influences sperm storage in the simultaneously hermaphroditic snail Arianta arbustorum. Invertebr Reprod Dev 49:93-101

Curril IM, LaMunyon CW (2006) Sperm storage and arrangement within females of the arctiid moth Utetheisa ornatrix. J Insect Physiol 52:1182-1188

Eberhard WG (1996) Female control: Sexual selection by cryptic female choice. Princeton University Press, Princeton

Hosken DJ, Meyer E, Ward PI (1999) Internal female reproductive anatomy and genitalic interactions during copula in the yellow dung fly, Scathophaga stercoraria (Diptera, Scathophagidae). Can J Zool 77:1975-1983

Hosken DJ, Ward PI (2000) Copula in yellow dung flies (Scathophaga stercoraria): investigating sperm competition models by histological examination. J Insect Physiol 46:1355-1363
Jann P, Blanckenhorn WU, Ward PI (2000) Temporal and microspatial variation in the intensities of natural and sexual selection in the yellow dung fly Scathophaga stercoraria. J Evol Biol 13:927-938

Jann P, Ward PI (1999) Maternal effects and their consequences for offspring fitness in the yellow dung fly. Funct Ecol 13:51-58

Kruuk LE (2004) Estimating genetic parameters in natural populations using the 'animal model'. Philos Trans R Soc B 359:873890

Lynch M, Walsh B (1998) Genetics and analysis of quantitative traits. Sinauer, Sunderland

Otronen M, Reguera P, Ward PI (1997) Sperm storage in the yellow dung fly Scathophaga stercoraria: identifying the sperm of competing males in separate female spermathecae. Ethology 103:844-854

Parker GA (1970) Sperm competition and its evolutionary consequences in insects. Biol Rev 45:525-567

Parker GA (1978) Searching for mates. In: Krebs JR, Davies NB (eds) Behavioural ecology : an evolutionary approach, 1st edn. Blackwell, Oxford, p 214

Rice WR, Steward AD, Morrow EH et al (2006) Assessing sexual conflict in the Drosophila melanogaster model system. Philos Trans R Soc B 361:287-299

Roff DA, Tucker J, Stirling G, Fairbairn DJ (1999) The evolution of threshold traits: effects of selection on fecundity and correlated response in wing dimorphism in the sand cricket. J Evol Biol 12:535-546

Ward PI (1998) A possible explanation for cryptic female choice in the yellow dung fly, Scathophaga stercoraria (L.). Ethology 104:97-110

Ward PI (2000) Cryptic female choice in the yellow dung fly Scathophaga stercoraria (L.). Evolution 54:1680-1686

Ward PI (in press) Post-copulatory selection in the yellow dung fly Scathophaga stercoraria (L.) and the mate-now-choose-later mechanism of cryptic female choice. Adv Stud Behav

Ward PI, Vonwil J, Scholte EJ, Knop E (2002) Field experiments on the distributions of eggs of different phosphoglucomutase (PGM) genotypes in the yellow dung fly Scathophaga stercoraria (L.). Mol Ecol 11:1781-1785

Zeh JA, Zeh DW (2001) Reproductive mode and the genetic benefits of polyandry. Anim Behav 61:1051-1063 